\title{
Human-Robot Cooperative Object Swinging
}

\author{
Philine Donner ${ }^{1}$, Alexander Mörtl ${ }^{2}$, Sandra Hirche $^{2}$ and Martin Buss ${ }^{1}$
}

\begin{abstract}
This paper investigates goal-directed cooperative object swinging as a novel physical human-robot interaction scenario. We develop an energy-based control concept, which enables a robot to cooperate with a human in a goal-directed swing-up task. The robot can be assigned to be a leader or an actively contributing follower. We conduct a virtual reality experiment to compare effort sharing and performance of mixed human-human and human-robot dyads. The leader and the follower controllers yield similar results compared to their human standard.
\end{abstract}

\section{INTRODUCTION}

With robots entering everyday life, cooperative tasks involving physical human robot interaction (pHRI) gain in importance. Collaborative object manipulation has attracted attention in the robotic community. Starting out with impedance based controllers [1], more sophisticated approaches have evolved that reduce the human effort in tasks like cooperative load transport [2]. Robots are no longer just passive helpers but active partners that understand human intentions [3], [4] and actively contribute to the task [5], [6]. Whereas most of the existing works on cooperative pHRI focus on quasistatic and pure translational object manipulation, only little attention has been paid to dynamic manipulation, such as swing motion. Imagine, for example, two persons cooperatively holding a sports mat or a large sack of potatoes, swinging it from side to side and releasing it at the same time in order to lift it onto a trolley. In the same way, dynamic swing motion may enable human-robot teams to manipulate larger and heavier objects than they normally would be able to manipulate on their own or through pure transfer motion (see Fig. 1).

Brachiating robots [7] and underactuated cable-suspended robots [8] are example applications for swing motion. A twolink robot is controlled to brachiate in a pendulum fashion like an ape by controlling it to a desired target dynamic in [7]. In [8] a three degrees-of-freedom parallel cable-driven robot, similar to the underactuated mechanism presented in this paper, is excited to reach a number of target points in a large workspace. In the area of pHRI, rope turning tasks have been investigated [9], [10]. While these works achieve continuous turning, even in the more recent work [9], the human still needs to take over the swing-up phase until a stable rope turning motion can be achieved by the robot.

\footnotetext{
${ }^{1}$ P. Donner and M. Buss are with the Institute of Automatic Control Engineering, Technische Universität München, 80290 München, Germany, \{philine.donner, mb\}@tum.de

${ }^{2}$ A. Mörtl and S. Hirche are with the Institute for Information-oriented Control, Technische Universität München, 80290 München, Germany, \{moertl, hirche\}etum. de
}

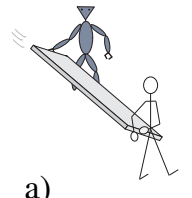

a)

Fig. 1. Flexible object swinging (b) interpreted as a mixture of rigid object swinging (a) and pendulum swinging (c).

As during cooperative load transport, information as well as energy is transferred through the mutual physical coupling to the object, which poses an additional challenge [3]. The haptic communication channel established through the coupling is known to enable fast and non-verbal negotiation of the physical effort between cooperating human dyads, resulting in temporally consistent interaction patterns [11]. These patterns of effort contribution can be also understood as a situation-dependent allocation of leader and follower roles, which are continuously blended between each other [6]. Alternatively, human haptic feedback interpreted as an agreement can equip robotic partners to dynamically allocate its role behavior [5].

In this work, we investigate cooperative object swinging by a robotic and a human partner. Through the swinging motion, the energy contained in the object is continuously increased up to a desired energy level. Similar continuous energy injection has also been used for brachiating robots [7] or to bring a cart-pendulum system from its stable equilibrium point to its unstable equilibrium point [12]. In our case, the desired energy level resembles a desired object height, at which the object could be released to perform a goaldirected throw. To the best of the authors' knowledge, no similar pHRI task has been investigated and implemented to date. Figure 1 shows how the swinging of semi-rigid objects (b) can be classified as a combination of two extremes: an oscillating entity formed through the partner's arms and a rigid object (a) and a simple pendulum that can oscillate itself (c). In this paper, we consider the latter, since oscillatory objects can be swung-up also with moderate actuation capabilities of a robotic partner limited in speed and amplitude. Important aspects as the repetitive movements used to incrementally increase the objects energy are preserved. The forces exerted by the partner can only indirectly be felt through the object dynamics, posing an additional challenge.

We base our control concept on the swing-up control method proposed in [12]. In particular, the principle of continuously pumping energy into the object is developed further to allow a leading robot to cooperatively swing up the 


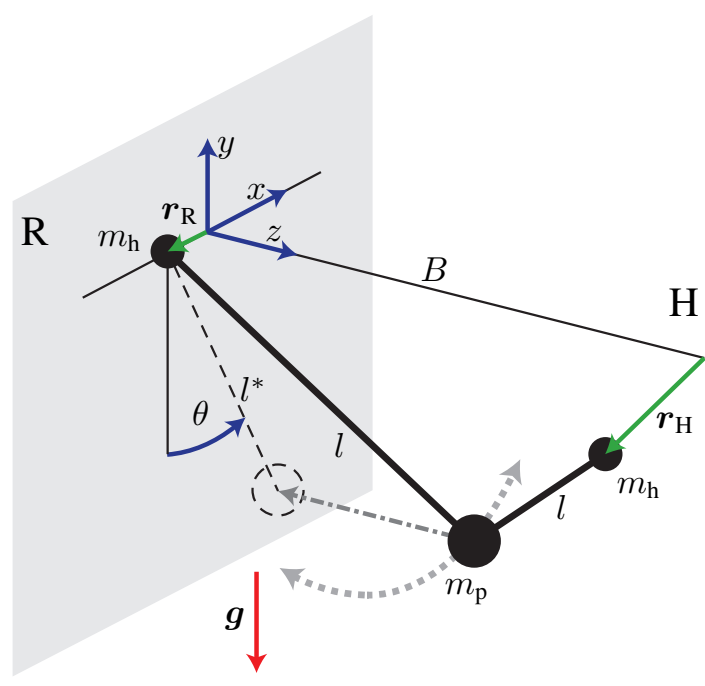

Fig. 2. V-pendulum setup: pendulum mass $m_{\mathrm{p}}$ actuated by a human $\mathrm{H}$ and robot $\mathrm{R}$ through the handle masses $m_{\mathrm{h}}$.

oscillatory object to a known desired energy level together with a human partner. The presented control concept is also shown to enable a following robot to actively contribute to the task even without knowing the human's desired energy level. In both cases, the robot accomplishes the swing-up task relying solely on haptic feedback from its endeffector.

The remainder of the paper is organized as follows. In Sec. II we model the object swinging task and develop two control laws: one that renders a robotic leader and one that renders a robotic follower. Sec. III presents the setup and design of a virtual reality experiment and introduces the applied evaluation criteria. After reporting and discussing our experimental results in Sec. IV, we draw our conclusion in Sec. V.

\section{ENERGY-BASED CONTROL}

\section{A. The v-pendulum setup}

In order to be able to focus on the cooperative swinging, we use a simple, oscillatory object as displayed in Fig. 2. We refer to this underactuated mechanism as a v-pendulum throughout this paper. The pendulum mass $m_{\mathrm{p}}$ is suspended from two ropes, both of length $l$. The ropes themselves are connected to handles of mass $m_{\mathrm{h}}$. The handle on the robot side $\mathrm{R}$ is restricted to movements in the $x$-direction in a limited range. Even though humans may move the handle in $3 \mathrm{D}$ on the human side $\mathrm{H}$ when swinging up the $\mathrm{v}$-pendulum, we choose to limit the robot's movement to the $x$-axis for simplicity and as a minimum requirement. The distance between the two handles in their initial configuration is $B$. Note that this distance changes when the two partners move independently. The v-pendulum is projected into the $x y$-plane on the robot side $\mathrm{R}$ as indicated by the dashdotted arrow. The resulting simple pendulum of length $l^{*}$ and deflection angle $\theta$ is shown by the dashed line. The position of the human and robot handles, with respect to their initial positions, are given by the vectors $\boldsymbol{r}_{\mathrm{H}}$ and $\boldsymbol{r}_{\mathrm{R}}$, respectively.

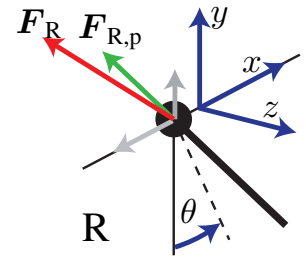

Fig. 3. Forces acting on the robot side of the v-pendulum.

We want the robot to swing the pendulum up solely with the help of haptic feedback. Figure 3 shows the forces acting on the robot side of the v-pendulum. We assume that the total force applied by the robot $\boldsymbol{F}_{\mathrm{R}}$ can be measured by a force sensor. By dynamically compensating for the forces caused by the handle mass $m_{\mathrm{h}}$, we obtain the force $\boldsymbol{F}_{\mathrm{R}, \mathrm{p}}$ exerted by the robot on the pendulum mass $m_{\mathrm{p}}$. Knowing $\boldsymbol{F}_{\mathrm{R}, \mathrm{p}}$ allows the computation of the projected deflection angle $\theta$

$$
\theta=\arctan \left(\frac{-F_{\mathrm{R}, \mathrm{p}, x}}{F_{\mathrm{R}, \mathrm{p}, y}}\right)
$$

and the position of the pendulum mass $m_{\mathrm{p}}$, with respect to the robot handle

$$
\boldsymbol{r}_{\mathrm{R}-\mathrm{p}}=-\frac{\boldsymbol{F}_{\mathrm{R}, \mathrm{p}}}{\left\|\boldsymbol{F}_{\mathrm{R}, \mathrm{p}}\right\|_{2}} l .
$$

\section{B. Robot as leader: known target deflection angle $\theta^{d}$}

First, we design a swing-up control that enables the robot to increase the energy contained in the v-pendulum until a target deflection angle $\theta^{\mathrm{d}}$ is reached. This target deflection angle is known to the robot. The robot takes the role of the leader, as it does not react to the human, but only urges to achieve the assigned goal.

Although the v-pendulum is more complex due to its twosided actuation, we show that the swing-up control for a simple inverted pendulum by [12] can be applied using the projection of the v-pendulum into the $x y$-plane (Fig. 2). We choose to base our control on this specific swing-up control for the following reasons:

- The swing-up controller is based on reaching a desired energy level, which can be easily adapted to achieve target angles in the range of $-\pi / 2<\theta^{\mathrm{d}}<\pi / 2$, as desired for our task.

- The pendulum is swung up through linear acceleration of an effector that obeys a restricted workspace.

- The low-pass characteristics of the approach make it robust to noise.

The equation of motion that describes the deflection angle of a simple inverted pendulum is given by

$$
\ddot{\theta}=-\frac{g}{l^{*}} \sin (\theta)-\frac{1}{l^{*}} \cos (\theta) \ddot{r}_{\mathrm{R}, x}
$$

with parameters and variables as specified in Fig. 2 and $\boldsymbol{r}_{\mathrm{R}}=$ $\left[\begin{array}{lll}r_{\mathrm{R}, x} & 0 & 0\end{array}\right]^{T}$. Note that for our v-pendulum this equation of motion only holds for the following restricted mo- 


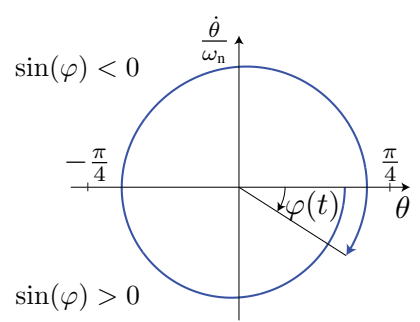

Fig. 4. Section of the phase portrait of a v-pendulum during swing-up. The normalized first time derivative of the deflection angle $\frac{\dot{\theta}}{\omega_{\mathrm{n}}}$ is plotted over the deflection angle $\theta$. With $\cos (\theta)>0$ for angles $-\frac{\pi}{2}<\theta<\frac{\pi}{2}$, $\operatorname{sgn}(\sin (\varphi))=-\operatorname{sgn}(\dot{\theta} \cos (\theta))$ holds.

tion: $\ddot{r}_{\mathrm{H}, x}=\ddot{r}_{\mathrm{R}, x}, r_{\mathrm{H}, y}=r_{\mathrm{H}, z}=0$ and the same initial position $r_{\mathrm{H}, x, 0}=r_{\mathrm{R}, x, 0}=0$. However, as in our scenario the initial handle distance $B \gg\left\|r_{\mathrm{H} / \mathrm{R}}\right\|$, the projected deflection angles $\theta$ on the human and the robot sides are almost equal. Thus, through proper choice of the handle acceleration $\ddot{r}_{\mathrm{R}, x}$ we can influence $\theta$ (3), which in turn couples the robot to the human motion.

The kinetic and potential energy of the pendulum under above specified mirrored motion is

$$
\begin{gathered}
V_{\text {kin }}=\frac{1}{2} m_{\mathrm{p}}\left[\left(l^{*} \cos (\theta) \dot{\theta}+\dot{r}_{\mathrm{R}, x}\right)^{2}+\left(l^{*} \sin (\theta) \dot{\theta}\right)^{2}\right] \\
V_{\text {pot }}=m_{\mathrm{p}} g l^{*}(1-\cos (\theta)) .
\end{gathered}
$$

Taking the time derivative of the sum of the energies (4) and (5), while disregarding the energy induced by the handle motion $\left(\dot{r}_{\mathrm{R}, x}=0\right)$ and inserting (3) yields an approximation of the change of pendulum energy $V$ as given in [12]

$$
\dot{V} \simeq-m_{\mathrm{p}} l^{*} \dot{\theta} \cos (\theta) \ddot{r}_{\mathrm{R}, x} .
$$

Thus, the pendulum energy will increase as long as $\operatorname{sgn}\left(\ddot{r}_{\mathrm{R}, x}\right)=-\operatorname{sgn}(\dot{\theta} \cos (\theta))$ holds.

The need to periodically excite underactuated suspended mechanisms close to its natural frequency is affirmed by [8]. Figure 4 shows a phase portrait for the v-pendulum. The inscribed angle $\varphi$ in the phase portrait approximately relates to the natural frequency of the pendulum $\omega_{\mathrm{n}}^{\prime}$ as follows [12]

$$
\varphi(t) \simeq \omega_{\mathrm{n}}^{\prime} t+\varphi_{0} .
$$

Thus, by choosing $\varphi(t)$ to be the argument of a sinusoidal input trajectory $r_{\mathrm{R}, x}$, the pendulum is excited close to its natural frequency. The phase angle can be computed through $\varphi=\arctan \left(-\frac{\dot{\theta}}{\theta \omega_{n}}\right)$, with $\omega_{\mathrm{n}}=\sqrt{\frac{g}{l^{*}}}$ being the approximation of the natural frequency of the pendulum for small angles $\theta$.

In [12], the input $r_{\mathrm{R}, x}$ is obtained by feeding the reference input

$$
r_{\mathrm{R}, x}^{\mathrm{d}}=a \frac{1}{g\left(\omega_{\mathrm{n}}\right)} \sin \left(\varphi(t)-\pi+\phi\left(\omega_{\mathrm{n}}\right)\right) .
$$

through the transfer function

$$
G(s)=\frac{\Omega^{2}}{s^{2}+2 \zeta \Omega s+\Omega^{2}}
$$

where $a$ is an amplitude factor, $g\left(\omega_{\mathrm{n}}\right)$ and $\phi\left(\omega_{\mathrm{n}}\right)$ are the amplitude and phase shift of the transfer function $G(s)$ at $\omega_{\mathrm{n}}, \Omega=\frac{\omega_{\mathrm{n}}}{c_{0}}$, and $c_{0}$ and $\zeta$ are design variables.

The resulting actuator trajectory $r_{\mathrm{R}, x}$ is approximately

$$
r_{\mathrm{R}, x} \simeq a \frac{g\left(\omega_{\mathrm{n}}^{\prime}\right)}{g\left(\omega_{\mathrm{n}}\right)} \sin \left(\varphi(t)-\pi+\phi\left(\omega_{\mathrm{n}}\right)-\phi\left(\omega_{\mathrm{n}}^{\prime}\right)\right) .
$$

As Fig. 4 shows the sign relation $\operatorname{sgn}(\varphi)=$ $-\operatorname{sgn}(\dot{\theta} \cos (\theta))=\operatorname{sgn}\left(\ddot{r}_{\mathrm{R}, x}\right)$ holds for deflection angles $-\pi / 2<\theta<\pi / 2$. Thus, the actuator acceleration $\ddot{r}_{\mathrm{R}, x}$ satisfies the sign condition (6), and the energy of the pendulum will increase as long as $a>0$ holds.

The amplitude factor $a$ is chosen to increase linearly with the energy error $V^{\mathrm{d}}-V$ and a slope of $\frac{a_{L}}{b_{L}}$ while being saturated at $\pm a_{\mathrm{L}}$

$$
\mathcal{L}: \quad a= \begin{cases}a_{\mathrm{L}} \operatorname{sgn}\left(V^{\mathrm{d}}-V\right) & \text { if }\left|V^{\mathrm{d}}-V\right| \geq b_{\mathrm{L}} \\ \frac{a_{\mathrm{L}}}{b_{\mathrm{L}}}\left(V^{\mathrm{d}}-V\right) & \text { else. }\end{cases}
$$

The desired energy may represent a desired deflection angle $\theta^{\mathrm{d}}$ or a desired final height $h^{\mathrm{d}}$ of the pendulum mass

$$
V^{\mathrm{d}}=m_{\mathrm{p}} g l^{*}\left(1-\cos \left(\theta^{\mathrm{d}}\right)\right)=m_{\mathrm{p}} g h^{\mathrm{d}} .
$$

The presented approach renders a robotic leader: by feeding a reference input of the form (8) through a transfer function (9), we obtain the trajectory (10). A robot tracking this trajectory will swing up a pendulum attached to its end effector to a desired energy level (12).

As stated earlier, the derivation as presented here, only holds for a v-pendulum for the restricted, mirrored handle movements. However, the nature of the approach to base the argument of the sine on the current angle $\theta$ ((8) and Fig. 4) and the low-pass-filter (9), makes it insusceptible to small inprecisions caused by the more complex geometry of our object. As simulations and experiments show (Sec. III), the presented swing-up control allows a robot to swing up the v-pendulum also for 2D handle trajectories on the human side, which do not have to be in accordance with the robot control.

However, the target energy has to be known by the robot. In a following step, we want to enable the robot to actively contribute to the swing-up task, but without knowing the desired energy in advance.

\section{Robot as follower: unknown target deflection angle $\theta^{d}$}

Cooperation between partners can be achieved even if the task goal is not known to all agents, as it can be inferred from observed actions. The assistive behavior may range from simple gravity compensation to followers that take over almost the complete share of the effort required to fulfill the task [5]. For our swing-up task, we expect an actively contributing follower, who takes over a significant share of the task effort. We base our robot follower approach on energy monitoring and formulate the following for our 
control law: The robot should inject (release) energy into (from) the pendulum whenever the human injects (releases) energy into (from) the pendulum.

In order to mathematically formulate this control law, we analyze the system energy in the following. Due to the passive nature of the v-pendulum setup [13], energy can only be added to the system through the forces applied at the human $\boldsymbol{F}_{\mathrm{H}}$ and the robot $\boldsymbol{F}_{\mathrm{R}}$ actuator sides (see Fig. 2 and Fig. 3). As we are only interested in the pendulum energy and not the kinetic energy of the handle masses $m_{\mathrm{h}}$, we obtain the energy input to the pendulum by using the dynamically compensated forces $\boldsymbol{F}_{\mathrm{R}, \mathrm{p}}$ and $\boldsymbol{F}_{\mathrm{H}, \mathrm{p}}$

$$
\dot{V}_{\mathrm{in}}=\dot{\boldsymbol{r}}_{\mathrm{H}}^{T} \boldsymbol{F}_{\mathrm{H}, \mathrm{p}}+\dot{\boldsymbol{r}}_{\mathrm{R}}^{T} \boldsymbol{F}_{\mathrm{R}, \mathrm{p}} .
$$

The total energy contained in the nondissipative pendulum equates to

$$
V=V(t)=\int_{0}^{t} \dot{V}_{\text {in }} \mathrm{d} \tau+V(0) .
$$

The energy contained in the pendulum mass is given by

$$
V=m_{\mathrm{p}} g\left(l^{*}+r_{\mathrm{p}, y}\right)+\frac{1}{2} m_{\mathrm{p}} \dot{\boldsymbol{r}}_{\mathrm{p}}^{T} \dot{\boldsymbol{r}}_{\mathrm{p}}
$$

where $\boldsymbol{r}_{\mathrm{p}}=\boldsymbol{r}_{\mathrm{R}}+\boldsymbol{r}_{\mathrm{R}-\mathrm{p}}$ describes the position of the pendulum mass $m_{\mathrm{p}}$ with $\boldsymbol{r}_{\mathrm{R}-\mathrm{p}}$ according to (2).

Thus, we obtain the change in pendulum energy caused by the human $\dot{V}_{\mathrm{H} \text {,in }}$ through

$$
\dot{V}_{\mathrm{H}, \text { in }}=\frac{\mathrm{d}}{\mathrm{d} t} V-\dot{V}_{\mathrm{R}, \mathrm{in}} .
$$

By use of the same actuator trajectory $r_{\mathrm{R}, x}^{\mathrm{d}}$ (10), but with a modified amplitude factor $a$ depending on the computed human energy input $\dot{V}_{\mathrm{H}, \text { in }}$, we obtain an actively contributing follower. We choose the amplitude factor $a$ to switch between the three discrete values $a_{\mathrm{dis}}=\left\{-a_{\mathrm{F}}, 0, a_{\mathrm{F}}\right\}$ depending on the magnitude and the sign of the human energy input $\dot{V}_{\mathrm{H} \text {,in }}$

$$
\mathcal{F}: \quad a_{\text {dis }}= \begin{cases}a_{\mathrm{F}} \operatorname{sgn}\left(\dot{V}_{\mathrm{H}, \text { in }}\right) & \text { if }\left|\dot{V}_{\mathrm{H}, \text { in }}\right| \geq b_{\mathrm{F}} \\ 0 & \text { else, }\end{cases}
$$

where $a_{\mathrm{F}}$ determines the maximum amplitude and $b_{F}$ defines a neutral zone, in which the robot amplitude is controlled to zero. This choice of the amplitude factor $a$ is similar to the choice for a robot leader (11), with the difference of a neutral zone instead of a ramp connecting the two maximum values $\pm a_{\mathrm{L} / \mathrm{F}}$. We avoid abrupt switching and thus jumps in the acceleration of the endeffector $\ddot{r}_{x}$ through ramps as

$$
\dot{a}=\tau_{\mathrm{F}} \operatorname{sgn}\left(a_{\mathrm{dis}}-a\right) .
$$

The blending time constant $\tau_{\mathrm{F}}$ progressively changes the robot's amplitude, i.e., its contributed energy flow in the direction indicated by the human partner. The amplitude factor laws (17) and (18) render an effort role allocation based on the inferred human contribution similar to [5].

The two controllers presented in this section result in two distinct behaviors: a robotic leader and a robotic follower.

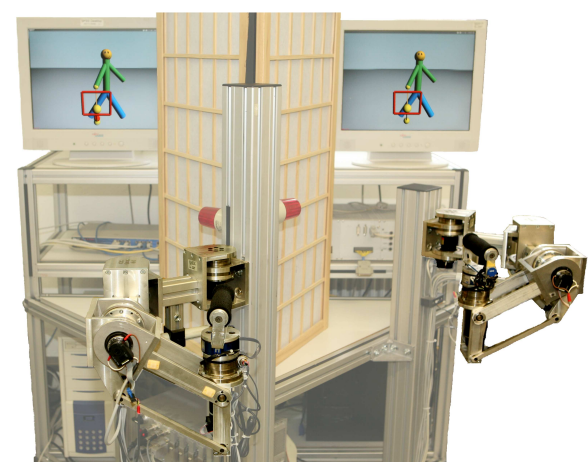

Fig. 5. Two 4 DoF haptic interfaces with handles and two screens render the virtual scene; one pair for each human partner. The participants conduct the cooperative task while standing in front of the devices.

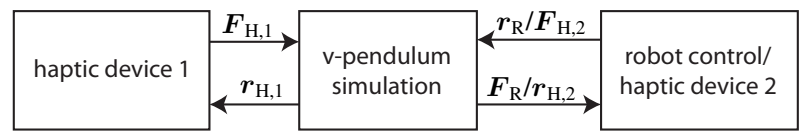

Fig. 6. Overall control structure for human-robot/human-human cooperative swing-up. The human side(s) of the v-pendulum are actuated through the forces $\boldsymbol{F}_{\mathrm{H}, 1 / 2}$, exerted during interaction with the haptic device. The robot control computes an actuation trajectory $\boldsymbol{r}_{\mathrm{R}}$, based on the measured force $\boldsymbol{F}_{\mathrm{R}}$

These distinct behaviors are realized through the choice of the amplitude factor $a$ as given by (11) and (17).

\section{EXPERIMENT}

In order to evaluate the proposed leader and follower control concept, we conducted a virtual reality experiment. Four right-handed participants ( 1 female, 3 male, age 2630) were told to swing up a v-shaped pendulum according to Fig. 2 in a virtual reality scene together with a human partner, with a virtual avatar and alone with one fixed end.

\section{A. Virtual reality rendering}

Both visual and haptic feedback is provided to the participants. The details on the rendering system are given in the following.

1) Hardware Setup: Two four degrees-of-freedom (DoF) manipulators provide high-fidelity haptic rendering to the human participants, see Fig. 5 for the experimental setup. The manipulators are computed-torque position controlled and have 6-DoF force/torque-sensors (JR3) attached to the handles. Two screens visualize the virtual reality scene for the participants. A partition between the screens prohibits the human participants to see the other person's screen.

2) Software Implementation: Both the simulation of the pendulum model and the control scheme of the haptic interfaces are implemented using MATLAB/Simulink Real-Time Workshop and executed at a sampling frequency of $1 \mathrm{kHz}$ on two personal computers running Linux real-time kernels. The control architecture for haptic rendering is shown in Fig. 6. The physical model of the pendulum is realized within the SimMechanics toolbox of MATLAB/Simulink through rigid bodies linked via unconstrained spherical joints. The 

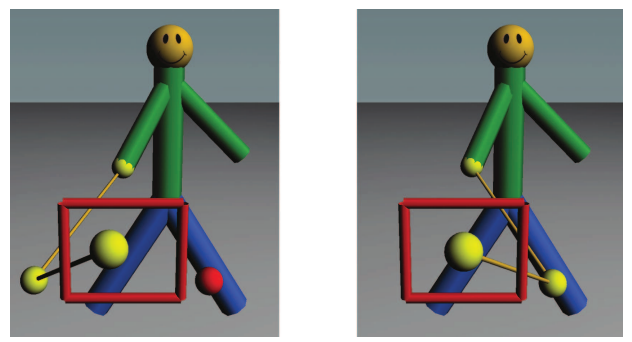

Fig. 7. Visualized scene for human-human cooperative swing-up. The red bounding boxes visualize the human workspace limits. The red sphere represents the goal position for the pendulum mass and is only displayed on the leader's screen.

rigid body assumption is valid, as the rope connecting both actuation bodies $m_{\mathrm{h}}$ and the pendulum mass $m_{\mathrm{p}}$ can be considered to be fully stretched during the complete swingup task. Whereas the robot only moves along the $x$-axis, we allow the human's effector to move within the $x y$-plane in a rectangular area of $0.24 \mathrm{~m} \times 0.18 \mathrm{~m}$ constrained by virtual walls. The $z$-axis is controlled to a constant position. This way the human feels the forces exerted by the pendulum in $x$ - and $y$-direction, which were found to be important for a realistic haptic perception of the pendulum state. The interaction of the human through the haptic interface with the virtual environment is realized by the Cartesian admittance control law

$$
F_{\mathrm{H}, x / y}=m_{\mathrm{h}} \ddot{r}_{\mathrm{H}, x / y}+d_{\mathrm{t}} \dot{r}_{\mathrm{H}, x / y}+F_{\mathrm{H}, \mathrm{p}, x / y}
$$

rendering a point-mass $m_{\mathrm{h}}$ with viscous damping $d_{\mathrm{t}}$ at the handle. For stability reasons a minimum damping $d_{\mathrm{t}}$ and a minimum mass $m_{\mathrm{h}}$ have to be assigned. This, in return, necessitates a relatively high pendulum mass for a sufficiently high ratio $\frac{m_{p}}{m_{h}}$. In order to decrease the gravitational load the human is exposed to, the handle mass $m_{\mathrm{h}}$ is gravity compensated. The parameters of the simulated v-pendulum are listed in Table I. A small, negligible inertia $\boldsymbol{I}_{\mathrm{p}}$ is assigned to the pendulum mass due to singularity issues within the SimMechanics model.

TABLE I

PARAMETERS OF THE PENDULUM SIMULATION

\begin{tabular}{ccccccc}
$m_{\mathrm{h}}[\mathrm{kg}]$ & $m_{\mathrm{p}}[\mathrm{kg}]$ & $\boldsymbol{I}_{\mathrm{p}}\left[\frac{\mathrm{kg}}{\mathrm{m}^{2}}\right]$ & $B[\mathrm{~m}]$ & $l[\mathrm{~m}]$ & $d_{\mathrm{r}}\left[\frac{\mathrm{Nms}}{\mathrm{deg}}\right]$ & $d_{\mathrm{t}}\left[\frac{\mathrm{Ns}}{\mathrm{m}}\right]$ \\
\hline 4 & 5 & $0.0005 \cdot \boldsymbol{I}$ & 1.32 & 0.9 & 0.0005 & 6
\end{tabular}

The v-pendulum as well as the virtual robot or human partner are visualized through an OpenGL-based implementation using the Visualization ToolKit. Figure 7 shows the displayed scene for human-human interaction. The control

TABLE II

CONTROL PARAMETERS

\begin{tabular}{ccccccc}
$c_{0}$ & $\zeta$ & $a_{\mathrm{L}}[\mathrm{m}]$ & $b_{\mathrm{L}}[\mathrm{J}]$ & $a_{\mathrm{F}}[\mathrm{m}]$ & $b_{\mathrm{F}}[\mathrm{W}]$ & $\tau_{\mathrm{F}}[\mathrm{s}]$ \\
\hline 0.9 & 1.2 & 0.04 & 2.93 & 0.036 & 0.58 & 1
\end{tabular}

parameters used for the robot leader and the robot follower are given in Table II. The two numerical differentiations in
(15) and (16) amplify noise, which we compensate for by passing $\dot{V}_{\mathrm{H} \text {,in }}$ through two consecutive moving average filters over 400 samples. The introduced delay is smaller than half a second and, thus, we expect the controller to react similarly fast as a human partner. However, model-based filters, e.g. Kalman filters, should be used in a real world experiment to avoid numerical differentiation of noisy force measurements $\left(\boldsymbol{F}_{\mathrm{R}}\right)$.

\section{B. Conditions and Procedure}

Each participant conducted three trials under the following experimental conditions in human interaction:

- HL-0, HL-HL, HL-HF, HF-HL,

and in human-robot interaction:

- HL-RL, HL-RF, HF-RL,

where HL (RL) and HF (RF) denotes a human (robot) leader and follower, respectively. Condition HL-0 required the human to swing up the pendulum alone while the other end of the pendulum was fixed. An extra trial in HL-0 was conducted before the actual experiment to allow the participants to get familiar with the task. The participants where informed about their roles HL or HF beforehand and asked to cooperate in order to achieve the joint goal: the energy level displayed by the goal sphere to the leader only.

The procedure was as follows: the human participant had to lift up the pendulum to the middle of the workspace. In case of an RL condition, the robot started with a small pointto-point movement to introduce slight swinging and, thus, a properly rotating phase angle $\varphi$ as needed for the robot control. Then the first goal sphere at $\boldsymbol{r}_{\mathrm{p}}^{\mathrm{d} 1}$ corresponding to a desired angle $\theta^{\mathrm{d} 1}=\pi / 6$ was displayed. After reaching the goal sphere once, the goal sphere stayed for another five pendulum periods. Then a new goal sphere at $\theta^{\mathrm{d} 2}=\pi / 4$ was displayed. After reaching that goal sphere and another five pendulum periods, the goal sphere was shifted back to angle zero. Thus, the participants were asked to completely release the pendulum energy.

\section{Measures}

The following measures are introduced to evaluate the above conditions. Note, that for each trial each measure is computed twice; once for each desired energy level.

1) Completion time $C T$ : The completion time is defined as the time span

$$
C T=t_{\mathrm{th}}-t_{\mathrm{id}}
$$

between the time of the first target hit $t_{\text {th }}$ $\left(\left\|\boldsymbol{r}_{\mathrm{p}}^{\mathrm{d}}-\boldsymbol{r}_{\mathrm{p}}\right\|_{2}<0.04 \mathrm{~m}\right)$ and the time of the initial display of the goal sphere $t_{\mathrm{id}}$.

2) Target hitting precision $T H_{r m s}$ : The target hitting precision is computed as the root-mean-square of the six dead points of the pendulum mass $\boldsymbol{r}_{\mathrm{p}, \mathrm{dp}}$ on the side of the goal sphere occurring after $t_{\text {th }}$

$$
T H_{\mathrm{rms}}=\sqrt{\frac{1}{6} \sum_{i=1}^{6}\left\|\boldsymbol{r}_{\mathrm{p}, \mathrm{dp}, i}-\boldsymbol{r}_{\mathrm{p}, i}^{\mathrm{d}}\right\|_{2}}
$$




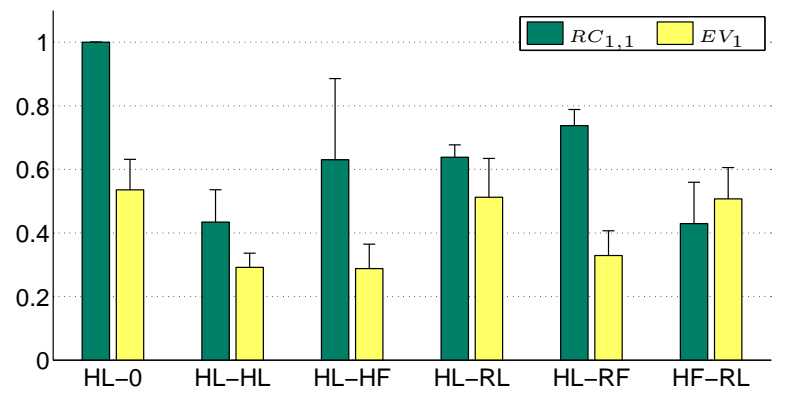

Fig. 8. Means and standard deviations of $R C_{1,1}$ and $E V_{1}$.

3) Relative contribution of the first agent $R C_{1}$ : The contribution of the first agent $R C_{1}$ is the energy input of the first agent in relation to the energy input of both agents during the time period $\left[t_{\mathrm{id}} t_{\mathrm{th}}\right]$ given by

$$
R C_{1}=\frac{V_{1}\left(t_{\mathrm{th}}\right)-V_{1}\left(t_{\mathrm{id}}\right)}{V_{1}\left(t_{\mathrm{th}}\right)-V_{1}\left(t_{\mathrm{id}}\right)+V_{2}\left(t_{\mathrm{th}}\right)-V_{2}\left(t_{\mathrm{id}}\right)} .
$$

Agent 1 is the left agent in the above nomenclature of conditions.

4) Energy efficiency $E V$ : The energy efficiency $E V$ penalizes energy flow waisted by the agents, i.e. any energy flow not directed towards the target energy level $V^{\mathrm{d}}$. We define

$$
E V=\frac{\left|V^{\mathrm{d}}-V\left(t_{\mathrm{id}}\right)\right|}{\int_{t_{\mathrm{id}}}^{t_{\mathrm{id}}}\left|\dot{V}_{1, \text { in }}\right|+\left|\dot{V}_{2, \text { in }}\right| \mathrm{d} \tau}
$$

where $t_{\mathrm{id} *}$ denotes the time when the next goal sphere is displayed. The energy efficiency $E V$ takes on values between 0 and 1 . Since the pendulum is slightly damped by a small $d_{\mathrm{r}}, E V=1$ is never reached. As we aim to penalize time periods in which an energy flow $\operatorname{sgn}\left(\dot{V}_{1, \text { in }}\right) \neq \operatorname{sgn}\left(\dot{V}_{2, \text { in }}\right)$ is voluntarily applied by the partners, we filter the energy flows with a third-order low-pass butterworth-filter with a cut-off frequency at $1 \mathrm{~Hz}$.

\section{Results AND Discussion}

For comparison between our six different conditions, we calculate the above four measures over all trials and for each condition and target angle separately. For the sake of brevity, we focus on the results for the desired angle $\theta^{\mathrm{d} 1}$ in detail.

\section{A. Analysis of the energy effort}

First, we analyze the energy flows into the system, and thus, the effort taken by the partners. Figure 8 shows the resulting energy efficiency $E V_{1}$ and the contribution of the first agent $R C_{1,1}$. The highest efficiency values are achieved for the conditions with a robotic leader. This is due to the perfectly monotonic energy flow produced by the leader controller. The energy efficiency for a robotic follower is in the same range as for the human-human combinations. Note that a high efficiency value for the HL-0 condition is obtained, since there is no partner interaction causing efficiency losses.

The contribution of the first agent $R C_{1,1}$ evaluates the target-directed energy input into the pendulum. Figure 8

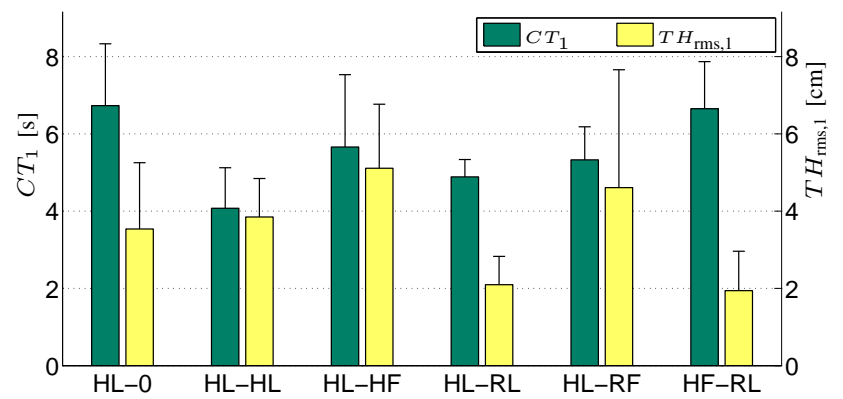

Fig. 9. Means and standard deviations of $C T_{1}$ and $T H_{\mathrm{rms}, 1}$.

suggests that for the conditions with a distinct human leader, i.e., HL-HF and HL-RF, the leader contributes more than half of the total energy needed to reach the goal. The relatively low contribution of the robotic agents is due to the rather conservative parameterization of the robot controllers with low amplitude factors $a_{\mathrm{L}}$ and $a_{\mathrm{F}}$. For the reported parameterization, the contribution of a robotic follower is comparable to the contribution of a human follower, both cooperating with a human leader. The standard deviation for the HL-HF condition is notably larger, due to the individual behavior of the participants, compared to the constant behavior of the robot follower.

\section{B. Analysis of the task performance}

Given the task of swinging up the pendulum to coincide with a displayed goal sphere, the completion time $C T_{1}$ and target hitting precision $T H_{\text {rms, } 1}$ shown in Fig. 9 evaluate the task performance. As expected, $C T_{1}$ is the smallest if both cooperating agents lead and, thus, know the desired angle. A robot follower as well as a human follower tend to decrease the completion time, compared to the human single performance. The relatively high completion time for the condition RL-HF is again a result of the conservative control parameterization.

The conditions involving a robot leader cause the best target hitting precision $T H_{\mathrm{rms}, 1}$. The good precision originates in the robot control, which continuously tracks the pendulum energy. In contrast, humans are expected to rely to a high extend on their visual feedback. The precision similarly decreases for a human leader cooperating with a robot or a human follower. However, the high standard deviation for the HL-RF condition stands out and is analyzed in the following by means of an example trial.

\section{Analysis of the robot follower}

A complete example trial for the HL-RF condition is given in Fig. 10. It shows the energy contribution of the human leader $V_{\mathrm{H} \text {,in }}$ and the robot follower $V_{\mathrm{R} \text {,in }}$, which sum up to the total energy input to the pendulum $V_{\text {in }}$. The total energy contained in the system is given by $V$, which is slightly lower than $V_{\text {in }}$ due to the damping $d_{\mathrm{r}}$ in the pendulum joints. The desired energy $V^{\mathrm{d}}$ is computed from the sequence of desired angles $\theta^{\mathrm{d} i}$. 


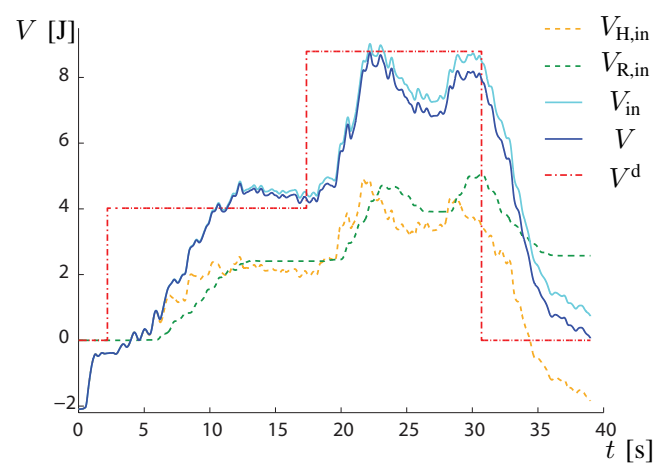

Fig. 10. Energy over time for a sample trial under HL-RF condition.

The trial starts as the human leader lifts the handle to the workspace middle, followed by the display of the first goal sphere at $\theta^{\mathrm{d} 1}=\pi / 6$. The robot is controlled to monotonically raise the energy level according to the human input with a short time delay. Once the target angle is reached, the human stops inserting more energy into the system, which causes the robot controller to enter the neutral zone, controlling its amplitude to zero (17). The measures target hitting precision $T H_{\mathrm{rms}, 1}=0.023 \mathrm{~m}$ and energy efficiency $E V_{1}=0.43$ for the first energy level in this trial are especially good compared to the means over all trials (see Fig. 8 and Fig. 9 ). The reason for the worse performance during other trials can be found when observing the next phase defined by the target angle $\theta^{\mathrm{d} 2}=\pi / 4$. The higher energy level is harder to be tracked by the human. As a result, the robot controller does not stay in its neutral zone, which in return makes it more difficult for the human to track the target energy level. As a consequence the measures for the second phase degrade to $T H_{\text {rms }, 2}=0.036 \mathrm{~m}$ and $E V_{2}=0.31$. Other participants tend to highly oscillate also around the lower energy level, which explains the high standard deviation of $T H_{\mathrm{rms}, 1}$ for the HL-RF conditions. This issue suggests that control parameters adapted to the individual human behavior may improve performance. Furthermore, a robot follower taking into account the magnitude of the human energy flow is expected to be beneficial.

\section{CONCLUSION AND FUTURE WORK}

In this work, we investigate goal-directed cooperative object swinging as a pHRI task. Cooperative swinging may enable human-robot teams to extend their object manipulation capabilities beyond quasi-static object transport. The joint swing-up of a v-shaped pendulum is investigated as a simplified example of a cooperative task. We present a control design that enables a robot to swing up the vpendulum in cooperation with a human. The robot can take on the two distinct roles of a leader and a follower. The robot follower actively contributes to the pendulum swingup, without knowing the task goal: a desired pendulum deflection angle. A virtual reality experiment serves as a proof of concept. The results reveal that the robot leader as well as the robot follower show comparable performance to a human leader and a human follower. We give directions on how the robot follower control performance could be further improved, as our experiment indicates a dependency on the human partner's swing-up behavior. In further research we will investigate how the swing-up for the simple v-pendulum can be extended to more complex oscillating objects as well as to the swing-up of rigid objects.

\section{ACKNOWLEDGMENT}

The authors would like to thank J. Bernhard, G. Goller, M. Karmann and F. Eutermoser for their support with setting up the experiments. This work is supported in part within the ERC Advanced Grant SHRINE Agreement No. 267877 (www.shrine-project.eu) and within the DFG excellence initiative research cluster Cognition for Technical Systems - CoTeSys (www. cotesys.org).

\section{REFERENCES}

[1] K. Kosuge, H. Yoshida, and T. Fukuda, "Dynamic control for robothuman collaboration," in Proc. 2nd IEEE Int. Workshop on Robot and Human Communication, 1993, pp. 398-401.

[2] B. Corteville, E. Aertbelien, H. Bruyninckx, J. De Schutter, and H. Van Brussel, "Human-inspired robot assistant for fast point-to-point movements," in Proc. IEEE ICRA, 2007, pp. 3639-3644.

[3] J. Medina, M. Lawitzky, A. Mörtl, D. Lee, and S. Hirche, "An experience-driven robotic assistant acquiring human knowledge to improve haptic cooperation," in Proc. IEEE/RSJ IROS, 2011, pp. 2416-2422.

[4] V. Fernandez, C. Balaguer, D. Blanco, and M. Salichs, "Active humanmobile manipulator cooperation through intention recognition," in Proc. IEEE ICRA, vol. 3, 2001, pp. 2668-2673.

[5] A. Mörtl, M. Lawitzky, A. Küçükyılmaz, M. Sezgin, C. Basdogan, and S. Hirche, "The Role of Roles: Physical Cooperation between Humans and Robots," Int. Journal of Robotics Research, vol. 31, no. 13, pp. 1656-1674, 2012.

[6] P. Evrard and A. Kheddar, "Homotopy switching model for dyad haptic interaction in physical collaborative tasks," in Proc. EHS EuroHaptics, 2009, pp. 45-50.

[7] J. Nakanishi, T. Fukuda, and D. Koditschek, "A brachiating robot controller," IEEE Trans. on Robotics and Automation, vol. 16, no. 2, pp. 109-123, 2000.

[8] N. Zoso and C. Gosselin, "Point-to-point motion planning of a parallel 3-dof underactuated cable-suspended robot," in Proc. IEEE ICRA, may 2012, pp. 2325-2330.

[9] C. Kim, K. Yonekura, H. Tsujino, and S. Sugano, "Physical control of the rotation center of an unsupported object rope turning by a humanoid robot," in Proc. 9th IEEE-RAS Int. Conf. on Humanoid Robots, 2009, pp. 148-153.

[10] Y. Maeda, A. Takahashi, T. Hara, and T. Arai, "Human-robot cooperation with mechanical interaction based on rhythm entrainmentrealization of cooperative rope turning," in Proc. IEEE ICRA, vol. 4, 2001, pp. 3477-3482 vol.4.

[11] K. Reed and M. Peshkin, "Physical Collaboration of Human-Human and Human-Robot Teams," IEEE Trans. Haptics, vol. 1, no. 2, pp. 108-120, 2008.

[12] K. Yoshida, "Swing-up control of an inverted pendulum by energybased methods," in Proc. ACC, vol. 6, 1999, pp. 4045-4047 vol.6.

[13] R. Lozano, I. Fantoni, and D. Block, "Stabilization of the inverted pendulum around its homoclinic orbit," Systems and Control Letters, vol. 40, no. 3, pp. 197-204, 2000. 